\title{
Freqüência horária e sazonalidade de Lutzomyia longipalpis (Diptera: Psychodidae: Phlebotominae) na Ilha de São Luís, Maranhão, Brasil
}

\author{
Hourly frequency and seasonality of Lutzomyia \\ longipalpis (Diptera: Psychodidae: Phlebotominae) \\ on São Luís Island, Maranhão, Brazil
}

José Manuel Macário Rebêlo 1

\footnotetext{
1 Núcleo de Patologia Tropical e Medicina Social, Departamento de Patologia, Universidade Federal do Maranhão. Praça Madre Deus 2, São Luís, MA 65025-560, Brasil. macariorebelo@uol.com.br
}

\begin{abstract}
This article presents the hourly and seasonal distribution of the Lutzomyia longipalpis sand fly captured on São Luís Island, Maranhão State. A total of 11,200 specimens were captured during monthly use of CDC light traps indoors and in animal sheds, between 6:00 PM and 6:00 AM, in 1996 and 1997. The sand fly behaved as an annual species, with a high frequency year-round, while it was most abundant during the rainy season (57.2\%) as compared to the dry season (42.8\%). The highest frequencies during the rainy season occurred in January and April and in the dry season in July and November. The species was found all night long, mainly between 6:00 PM and 10:00 PM in animal sheds and between 8:00 PM and 2:00 AM indoors.

Key words Phlebotominae; Psychodidae; Vector Ecology; Insect Vector

Resumo A distribuição sazonal e horária de Lutzomyia longipalpis foi estudada nos ambientes peri e intradomiciliar de duas localidades da Ilha de São Luís, estado do Maranhão. Os 11.200 exemplares capturados foram atraídos por quatro armadilhas CDC nos anos de 1996 e 1997, mensalmente, das $18 \mathrm{~h}$ às $6 \mathrm{~h}$. L. longipalpis comportou-se como uma espécie anual, tendo sido encontrada em alta freqüencia em todos os meses do ano, tendendo a ser mais abundante no período chuvoso $(57,2 \%)$ do que no seco $(42,8 \%)$. As maiores freqüências foram observadas nos meses de janeiro e abril, no período chuvoso, e em julho e novembro, no período seco. Foi encontrada a noite inteira, porém foi mais freqüente entre $18 \mathrm{~h}$ e $22 \mathrm{~h}$ no peridomicílio, e entre $20 \mathrm{~h}$ e $2 \mathrm{~h}$ no intradomicílio.

Palavras-chave Phlebotominae; Psychodidae; Ecologia de Vetores; Insetos Vetores
\end{abstract}




\section{Introdução}

Os estudos de Rebêlo et al. (1999a, 1999b) mostraram que Lutzomyia longipalpis, o vetor da leishmaniose visceral (calazar) no nordeste brasileiro, é uma das espécies mais comuns e a que melhor se adapta ao convívio com o homem e animais domésticos na Ilha de São Luís. A rápida adaptação desse vetor ao peridomicílio nas áreas rurais e, até mesmo, nos bairros periféricos dos grandes centros urbanos ocorreu em virtude da modificação progressiva da vegetação primitiva da Ilha, ao longo dos últimos vinte anos.

Os projetos de desenvolvimento associados ao deslocamento de grupos populacionais vindos do interior (zona rural) e de outros estados nordestinos para os centros urbanos da Ilha (São Luís, Raposa, São José de Ribamar e Paço do Lumiar) contribuíram para que essas alterações ambientais ocorressem (Costa et al., 1995; Nascimento et al., 1996).

A criação de aglomerados populacionais, sem a mínima infra-estrutura sanitária, precedeu à destruição dos ecótopos naturais do vetor, ficando as pessoas expostas aos fatores de risco responsáveis pela disseminação do calazar, como a proximidade com os reservatórios silvestres, onde o L. longipalpis passou a ser o elo entre as fontes de infecção, o homem e o cão doméstico.

Neste trabalho, pretende-se estudar a ocorrência sazonal e a freqüência horária de L. longipalpis nessas áreas modificadas na Ilha de São Luís. O intuito é obter informações que dêem subsídios para estabelecer medidas de controle desse importante inseto vetor.

\section{Material e métodos}

\section{Descrição da área}

O estudo foi realizado na Ilha de São Luís, situada no litoral setentrional brasileiro a 2032'LS e 44043'LW, com uma área de 905 km², e separada do continente pelo Estreito dos Mosquitos. É constituída por terras baixas, elevando-se a $32 \mathrm{~m}$ de altitude onde está localizada a cidade de São Luís, a capital do Estado do Maranhão. Limita-se, ao norte, com o Oceano Atlântico; a oeste, com a Baía de São Marcos; a leste, com as Baías de São José de Ribamar e do Arraial e, ao sul, com o Estreito dos Mosquitos (Figura 1).

Até os anos 60/70, grande parte da Ilha era coberta por mata latifoliada, babaçual (Attalaea phalerata) e floresta paludosa marítima (manguezal). Entretanto, nas últimas duas dé- cadas, a vegetação local sofreu profunda modificação por causa da demanda imobiliária e derrubadas constantes e desenfreadas das matas primitivas. Atualmente, na zona rural, predominam as capoeiras entremeadas por babaçu e manchas de cerrados. Ao longo dos rios, onde existem matas ciliares, predominam os buritizais (Mauritia vinifera) e juçarais (Euterpe oleraceae), enquanto, na zona do litoral, encontram-se as formações pioneiras (campo e restinga) e manchas de cerrado, além do manguezal.

O clima é o tropical semi-úmido, com uma estação chuvosa, de janeiro a junho, que concentra, em média, cerca de $94 \%$ do total anual das chuvas. A estação seca, de julho a dezembro, concentra apenas $6 \%$. O total pluviométrico é elevado, por volta de $1.900 \mathrm{~mm}$ anuais, em média. As temperaturas são elevadas durante o ano todo $\left(26^{\circ} \mathrm{C}\right.$, em média), com variação anual pequena (IBGE, 1984).

\section{Amostragem}

O estudo foi realizado em duas áreas rurais, uma de ocupação antiga (vinte anos) e outra de ocupação recente (dez anos), ambas situadas em áreas de capoeira. Os flebótomos foram capturados nos anos de 1996 e 1997, mensalmente, das $18 \mathrm{~h}$ às $6 \mathrm{~h}$, com uso de armadilhas luminosas do tipo CDC, nos ambientes peri e intradomiciliar. Em cada ambiente das duas áreas, foi instalada uma armadilha CDC a 1,5 $\mathrm{m}$ de altura, sempre no mesmo local, totalizando quatro armadilhas.

O intradomicílio foi representado pelo dormitório das habitações. Os abrigos de animais domésticos, até um raio de $20 \mathrm{~m}$ de distância, constituiu o peridomicílio. Cada armadilha funcionou 12 horas consecutivas, e, a cada hora, trocava-se o saco com o intuito de separar os espécimes por horário. Uma vez capturados, os exemplares eram mortos em câmaras de acetato de etila e, em seguida, fixados em álcool a $70 \%$; depois, eram transportados para o laboratório, onde parte deles era montada em Berlese.

Todos os espécimes capturados foram identificados no Laboratório de Entomologia da Universidade Federal do Maranhão (UFMA), pelos técnicos da UFMA/Fundação Nacional de Saúde (FNS), auxiliados pela chave de identificação proposta por Young \& Duncan (1994).

Utilizou-se teste estatístico não paramétrico (Qui-Quadrado) para analisar as diferenças entre as estações e horários. As diferenças foram consideradas significativas quando a probabilidade (p) do erro foi inferior a $5 \%(\mathrm{p}<0,05)$. O coeficiente de correlação ( $r$ ) entre o número 
de espécimes encontrados e as médias da temperatura, umidade relativa do ar e pluviosidade, medidas ao longo do ano, também foram utilizados.

\section{Resultados e discussão}

Os dados aqui apresentados mostram que milhares de "furrupas" e/ou "arrupiados", como são chamados os flebótomos na Ilha de São Luís, Maranhão, podem ser encontrados durante a noite, em todos os meses do ano, associados com animais domésticos e com o homem, nas suas habitações.

Nos dois anos, coletaram-se 11.200 espécimes de L. longipalpis, entre machos e fêmeas, constituindo-se na espécie mais abundante na Ilha de São Luís, sendo mais comum no peridomicílio (88\%) do que dentro das habitações (12\%).

\section{Flutuação sazonal}

Do total de espécimes amostrado, cerca de $57,2 \%$ (6.403) foram capturados no período chuvoso, contra $42,8 \%$ (4.797) no período seco. Essa diferença foi estatisticamente significativa $(\mathrm{p}<0,05)$. As maiores freqüências foram observadas nos meses de janeiro e abril, no período chuvoso, e em julho e novembro, no período seco (Figuras 2 e 3 ).

Observou-se, pelo teste de correlação, que a temperatura $(r=0,199)$, a umidade relativa do $\operatorname{ar}(r=0,009)$ e a pluviosidade $(r=0,375)$ não constituíram fatores de influência mensal na densidade de L. longipalpis. Todavia, quando esses fatores ambientais foram relacionados com a freqüência do flebótomo por estação, constatou-se que a pluviosidade exerceu forte influência em sua distribuição sazonal $(r=1)$.

Essa tendência de L. longipalpis predominar no período chuvoso já foi observada nas décadas de 50 e 60 nas áreas semi-áridas do nordeste brasileiro. Nas áreas de endemismo de calazar do Ceará (Deane, 1956) e da Bahia (Sherlock \& Guitton, 1969), as populações de $L$. longipalpis aumentam nas épocas de chuvas. No entanto, em outras áreas biogeográficas, esse padrão pode mudar. No leste da Costa Rica, área de clima quente, baixa precipitação pluvial e de endemismo de calazar (Zeledón et al., 1984), semelhante ao nordeste do Brasil, a maior abundância de L. longipalpis ocorre na estação seca.

Em áreas de focos de calazar, no Mato Grosso do Sul, os flebótomos ali encontrados tendem a apresentar picos na primavera, verão
Figura 1

Mapa da Ilha de São Luís - Maranhão, mostrando os quatro municípios e os pontos (1 e 2) de capturas de Lutzomyia longipalpis, nos anos de 1996/1997.
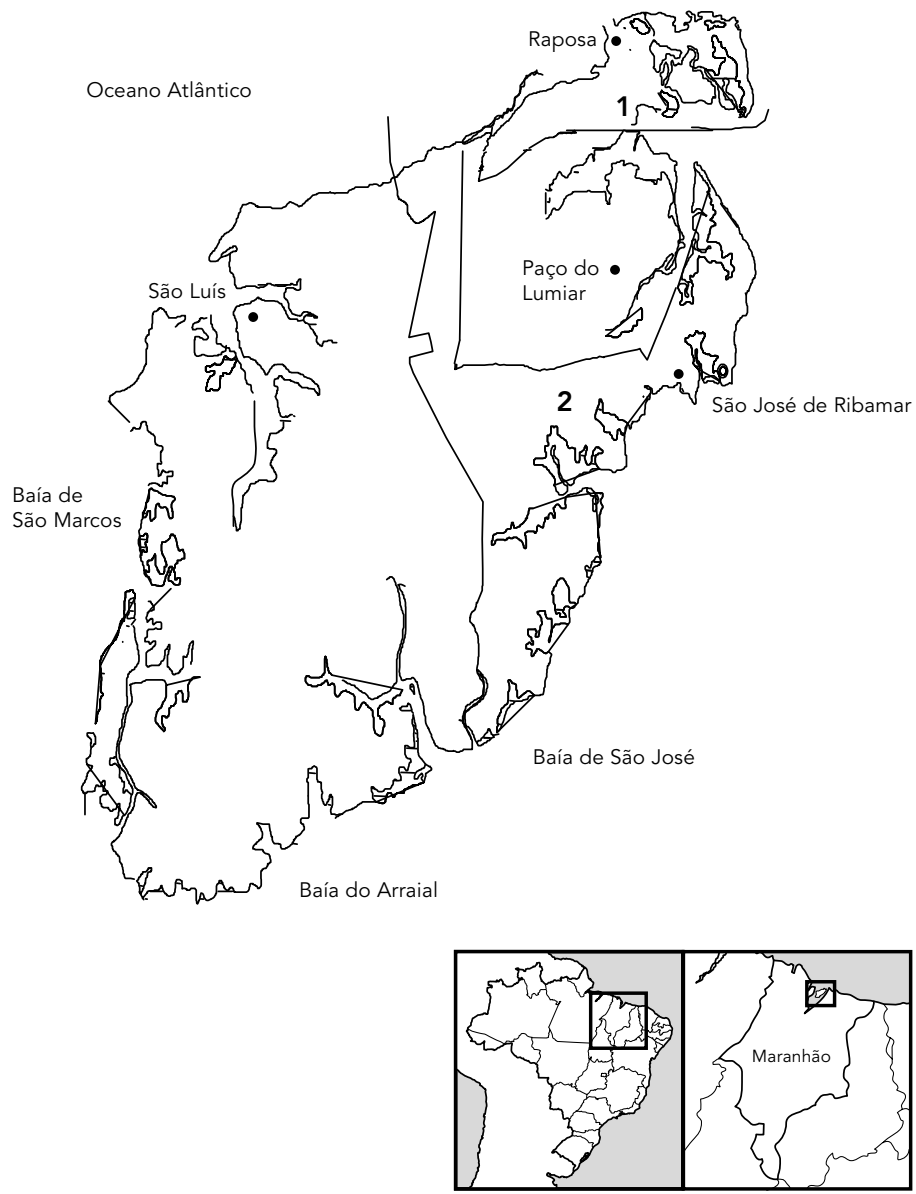

e outono, estações que representam os meses chuvosos e de temperatura mais elevada (Galati et al., 1997). Observa-se esse mesmo padrão nas áreas tropicais de altitude, onde geralmente a densidade dos flebótomos tende a aumentar nos meses mais quentes e úmidos do ano, que correspondem ao período chuvoso, como ocorre no Rio de Janeiro (Aguiar \& Soucasaux, 1984) e no Planalto Paulista (Barreto, 1943), podendo as menores freqüências ser observadas nos meses mais frios e secos (julho e agosto). No norte do Paraná, os picos de várias espécies de flebótomos foram registrados em março, abril e julho, e as depressões, na primavera (Teodoro et al., 1993), padrão um pouco diferente daqueles descritos no Rio de Janeiro e em São Paulo. 
Figura 2

Flutuação sazonal de Lutzomyia longipalpis. Ilha de São Luís, Maranhão, Brasil, 1996/1997.

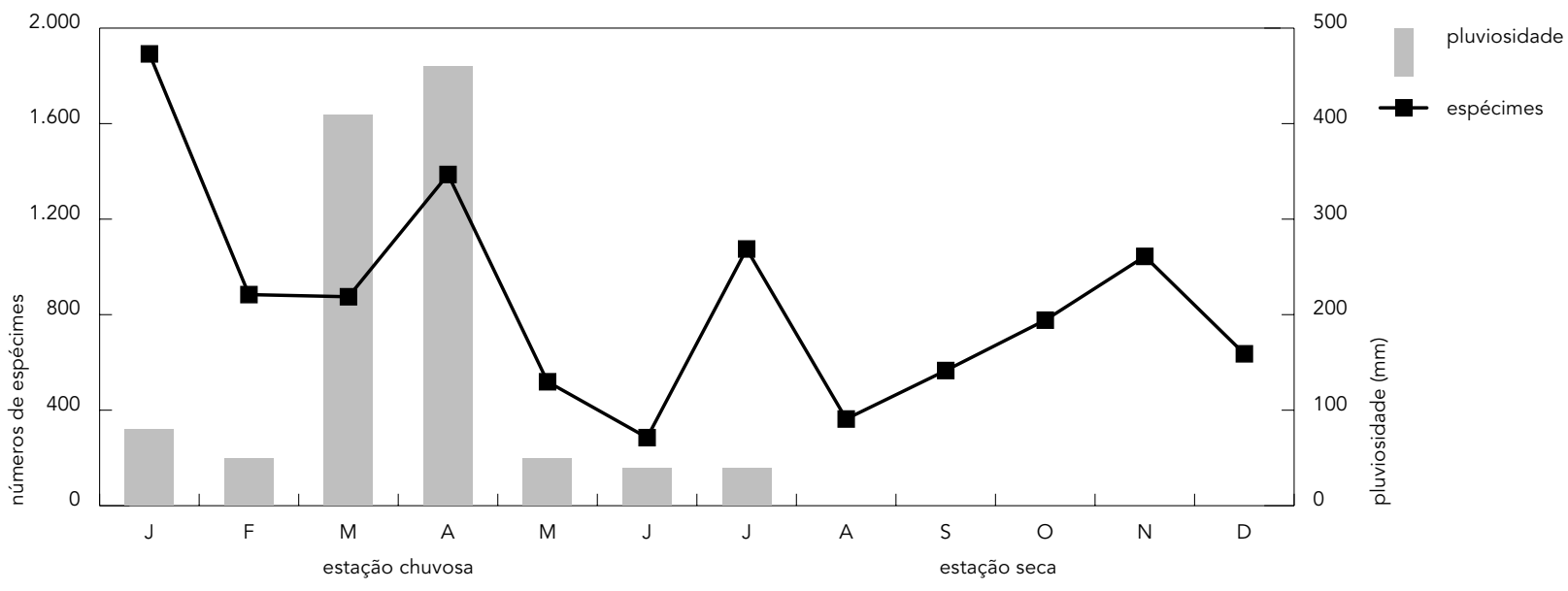

Figura 3

Variação da temperatura e da umidade relativa do ar. Ilha de São Luís, Maranhão, Brasil, 1996/1997.

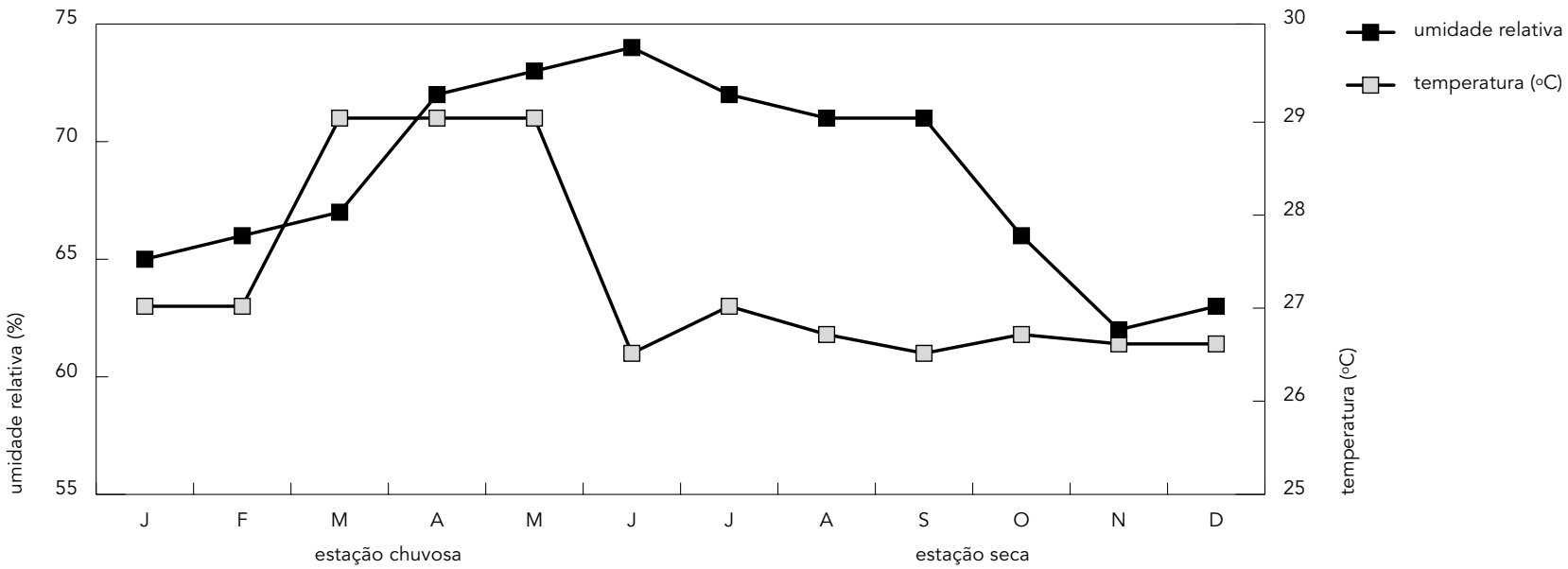

\section{Freqüência horária}

Neste trabalho, a atividade de L. longipalpis foi estudada apenas no período noturno (incluindo o crepúsculo vespertino e matutino), o qual vem sendo identificado pela população local, de áreas endêmicas de calazar, como aquele em que o flebótomo costuma picar as pessoas nas suas moradias e no ambiente peridomiciliar.
De um modo geral, o L. longipalpis distribuiu-se em todos os horários, tendendo a ocorrer com maior freqüência ao longo da noite do que no crepúsculo vespertino e matutino. Cerca de $60,8 \%$ dos espécimes foram capturados na primeira metade da noite, contra $39,2 \%$ na segunda metade. Essas diferenças foram significativas $(\mathrm{p}<0,05)$. A maior concentração de espécimes ocorreu entre 19h e 23h (Figura 4). Em ambos os ambientes, as freqüências fo- 
Freqüência horária de Lutzomyia longipalpis. Ilha de São Luís, Maranhão, Brasil, 1996/1997.

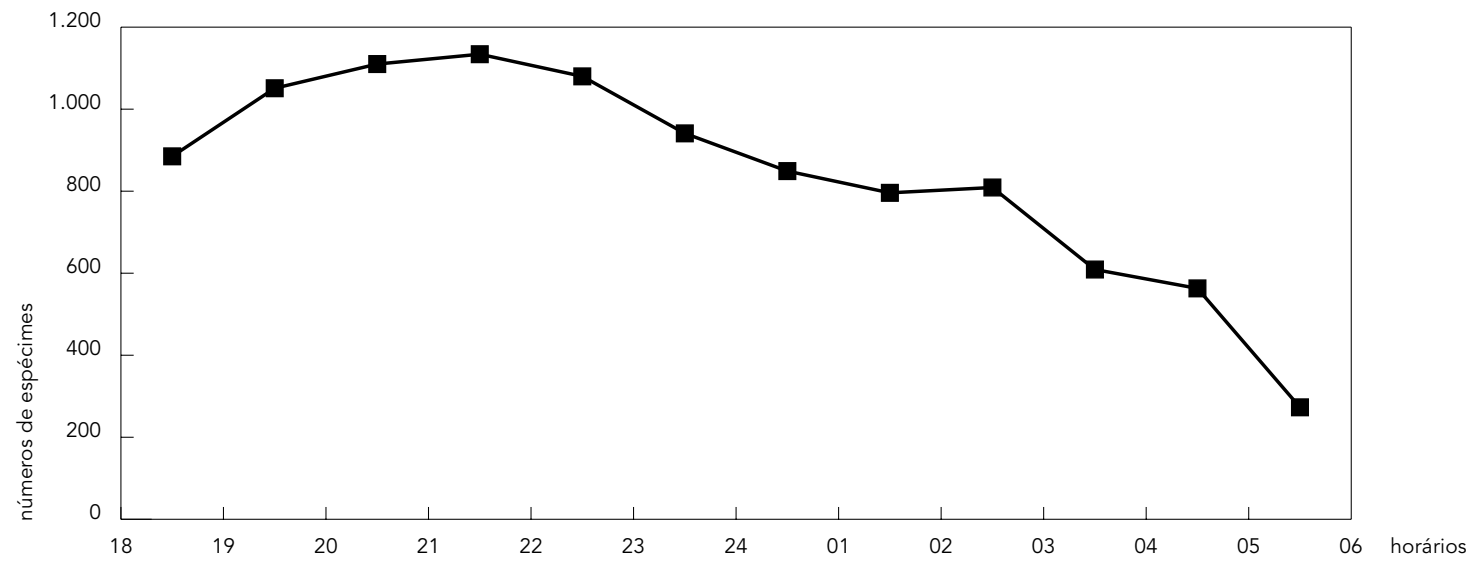

Figura 5

Freqüência horária de Lutzomyia longipalpis nos ambientes peri e intradomiciliar. Ilha de São Luís,

Maranhão, Brasil, 1996/1997.

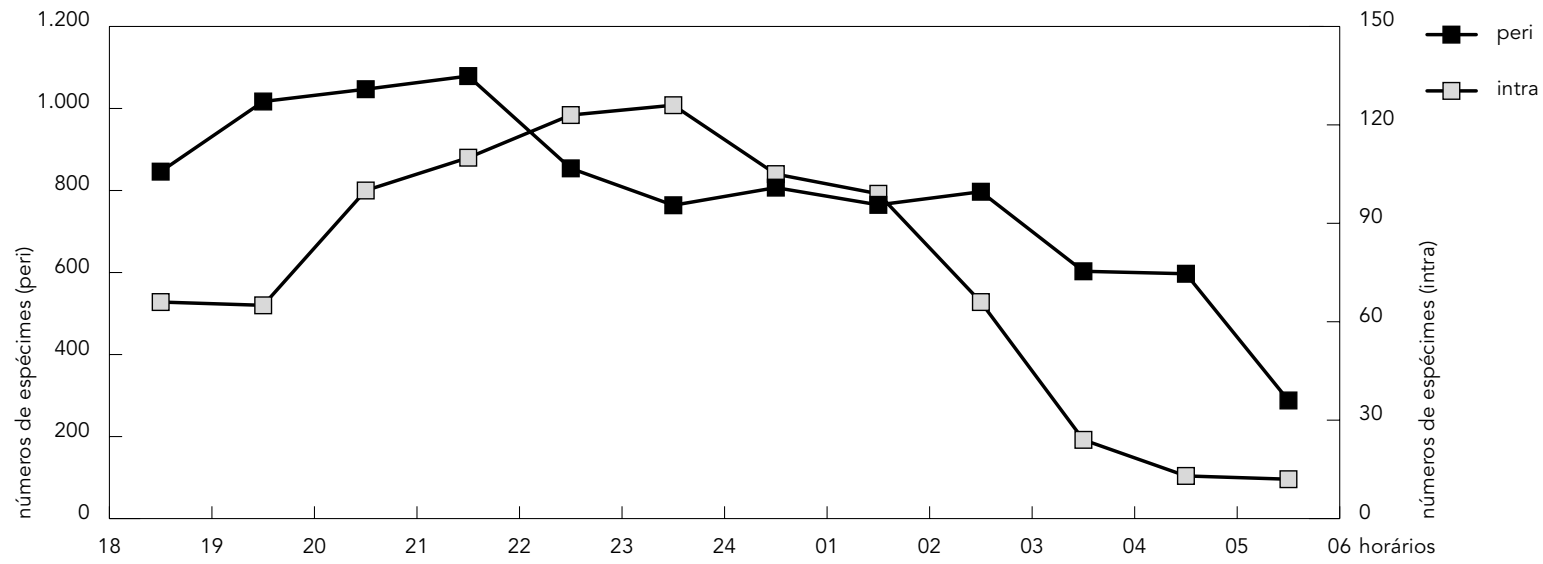

ram significativamente $(\mathrm{p}<0,05)$ maiores no crepúsculo vespertino (18h às 19h) do que no matutino (4h às 5h) (Figura 5).

As observações levadas a efeito no peridomicílio (galinheiro) mostraram maior freqüência do flebótomo entre $19 \mathrm{~h}$ e $22 \mathrm{~h}$. Essa predominância noturna foi também verificada em outros locais e ocasiões. Deane (1956), utilizando isca animal (eqüino), no Ceará, verificou que as fêmeas, apesar de entrarem em ativida- de durante o dia, tendem a apresentar maior densidade durante a noite, e, no peridomicílio, já foram observados picos das $18 \mathrm{~h}$ às $20 \mathrm{~h}$ (Deane \& Deane, 1962). Em observações realizadas na Ilha de Marajó, Estado do Pará, o rendimento das coletas realizadas fora da casa foi maior entre $19 \mathrm{~h}$ e $20 \mathrm{~h}$, e nos abrigos animais, entre 20h e 22h (Quinnell \& Dye, 1994). No México, em observações feitas com isca humana, a atividade de L. longipalpis iniciou-se por volta 
das $21 \mathrm{~h}$, sendo mais intensa entre $23 \mathrm{~h}$ e $4 \mathrm{~h}$, cessando às $6 \mathrm{~h}$ (Biagi et al., 1965). Na Costa Rica, Zeledón et al. (1984) capturaram uma grande quantidade de espécimes de L. longipalpis entre $19 \mathrm{~h}$ e $24 \mathrm{~h}$.

No ambiente intradomiciliar, de acordo com Deane (1956) e Alencar (1959), o "arrupiado" pode ser encontrado em qualquer hora do dia ou da noite. As nossas observações noturnas mostraram haver maior freqüência entre $20 \mathrm{~h}$ e $2 \mathrm{~h}$. No Ceará, as observações de Alencar apresentaram maior rendimento no horário compreendido entre $19 \mathrm{~h}$ e $20 \mathrm{~h}$, enquanto, na Bahia, horários de pico ocorreram mais tardiamente, entre $21 \mathrm{~h}$ e $23 \mathrm{~h}$ (Sherlock \& Guitton, 1969; Sherlock, 1996).

De um modo geral, pode-se perceber, comparando os nossos dados com os de outros autores, que L. longipalpis tende a ser muito abundante ao longo da noite, variando o pico de atividade de uma área para outra. Essas diferenças se devem a múltiplos fatores, incluindo condições locais da área, não apenas de ordem climáticas, como também do próprio comportamento das populações do flebótomo (Forattini, 1973). Acrescenta-se ainda o tipo de coleta (CDC, sucção nas paredes, sobre as pessoas etc.); o tipo de casa e a facilidade de acesso do flebótomo a ela e, entre outros, a época do ano.

Na zona rural da Ilha de São Luís, observase que L. longipalpis inicia sua atividade já com um pico na primeira hora da noite (19h) e é mais abundante no peridomicílio, ficando os animais domésticos mais expostos às suas investidas. Por outro lado, as habitações humanas dessas áreas, por apresentarem uma baixa qualidade, não oferecem uma proteção eficiente às pessoas, visto que os flebótomos podem ser encontrados nos dormitórios a noite toda, em qualquer época do ano, mas em menor freqüência do que no peridomicílio (Rebêlo, 1999a). A julgar pelos nossos resultados, os ataques mais intensos dentro dessas casas coincidem com os horários (20h às $24 \mathrm{~h}$ ) em que as pessoas estão repousando em seus aposentos.

\section{Referências}

ALENCAR, J. E., 1959. Calazar Canino. Contribuição para o Estudo da Epidemiologia do Calazar no Brasil. Fortaleza: Imprensa Oficial.

AGUIAR, G. M. \& SOUCASAUX, T., 1984. Aspectos da ecologia dos flebótomos do Parque Nacional da Serra dos Órgãos, Estado do Rio de Janeiro. I. Freqüência mensal em isca humana (Diptera, Psychodidae, Phlebotominae). Memórias do Instituto Oswaldo Cruz, 79:197-209.

BIAGI, A. M.; BIAGI, F. F. \& MILANARI, J. L., 1965. KalaAzar en México. Antropofilía y actividad horaria de Phlebotomus longipalpis Lutz y Neiva, 1912 (Diptera, Psychodidae). Revista del Instituto de Salubridades y Enfermedades Tropicales, 25:13-19.

BARRETTO, M. P., 1943. Observações sobre a Biologia, em Condições Naturais, dos Flebótomos do Estado de São Paulo (Diptera, Psychodidae). Tese de Livre-Docência, São Paulo: Faculdade de Medicina, Universidade de São Paulo.

COSTA, J. M. L.; VIANNA, G. L.; SALDANHA, A. R.; NASCIMENTO, M. D. S.; ALVIM, A. C.; BURATTINNI, M. N. \& SILVA, A. R., 1995. Leishmaniose visceral no Estado do Maranhão, Brasil. A evolução de uma epidemia. Cadernos de Saúde Pública, 11: 321-324. 
DEANE, L. M., 1956. Leishmaniose Visceral no Brasil. Estudos sobre Reservatórios e Transmissores no Estado do Ceará. Rio de Janeiro: Serviço Nacional de Educação Sanitária.

DEANE, L. M. \& DEANE, M. P., 1962. Visceral leishmaniasis in Brazil: Geographical distribution and transmission. Revista do Instituto de Medicina Tropical, 4:198-212.

FORATTINI, O. P., 1973. Entomologia Médica. v. 4, São Paulo: Editora Edgard Blücher/Edusp.

GALATI, E. A. B.; NUNES, V. L. B.; REGO Jr., F. A.; OSHIRO, E. T. \& CHANG, M. R., 1997. Estudo de flebotomíneos (Diptera, Psychodidae) em foco de leishmaniose visceral no Estado de Mato Grosso do Sul, Brasil. Revista de Saúde Pública, 31:378-390.

IBGE (Fundação Instituto Brasileiro de Geografia e Estatística), 1984. Atlas do Maranhão. Rio de Janeiro: IBGE/Governo do Estado do Maranhão.

NASCIMENTO, M. D. S. B.; COSTA, J. M. L.; FIORI, B. I. P. \& VIANA, G. M. C., 1996. Aspectos epidemiológicos determinantes na manutenção da leishmaniose visceral no Estado do Maranhão, Brasil. Revista da Sociedade Brasileira de Medicina Tropical, 29:233-240.

QUINNELL, R. J. \& DYE, C., 1994. An experimental study of the peridomestic distribution of Lutzomyia longipalpis (Diptera: Psychodidae). Bulletin of Entomological Research, 84:379-382.

REBÊLO, J. M. M.; ARAÚJO, J. C.; CARVALHO, M. L.; BARROS, V. L. L.; SILVA, F. S. \& OLIVEIRA, S. T., 1999a. Flebótomos (Lutzomyia, Phlebotominae) da Ilha de São Luís, zona do Golfão Maranhense, Brasil. Revista da Sociedade Brasileira de Medicina Tropical, 32:247-253.
REBÊLO, J. M. M.; LEONARDO, F. S.; COSTA, J. M. L.; PEREIRA, Y. N. O. \& SILVA, F. S., 1999b. Flebotomíneos (Diptera, Psychodidae) de área endêmica de leishmanioses na região dos cerrados, Estado do Maranhão, Brasil. Cadernos de Saúde Pública, 15: 623-630.

SHERLOCK, I. A., 1996. Ecological interactions of visceral leishmaniasis in Bahia. Memórias do Instituto Oswaldo Cruz, 91:671-683.

SHERLOCK, I. A. \& GUITTON, N., 1969. Observações sobre calazar em Jacobina, Bahia. IV. Variação horária e estacional de Phlebotomus longipalpis. Revista Brasileira de Malariologia, 21:715-727.

TEODORO, U.; SALVIA FILHO, V.; LIMA, E. M.; SPINOSA, R. P.; BARBOSA, O. C.; FERREIRA, M. E. M. C. \& SILVEIRA, T. G. V., 1993. Flebotomíneos em área de transmissão de leishmaniose tegumentar na Região Norte do Estado do Paraná-Brasil. Revista de Saúde Pública, 27:190-194.

ZELEDON, R.; MURILLO, J. \& GUTIERREZ, H., 1984. Observaciones sobre la ecología de Lutzomyia longipalpis (Lutz \& Neiva, 1912) y posibilidades de existencia de leishmaniasis visceral en Costa Rica. Memórias do Instituto Oswaldo Cruz, 79: 455-459.

YOUNG, D. G. \& DUNCAN, M. A., 1994. Guide to the Identification and Geographic Distribution of Lutzomyia Sand Flies in Mexico, the West Indies, Central and South America (Diptera: Psychodidae). Gainesville: American Entomological Institute. 